\title{
Implementing a Standing Order Immunization Policy: A Minimalist Intervention
}

\author{
George R. Gamble, PhD, Adam O. Goldstein, MD, and Rachel S. Bearman, MA
}

Introduction: Standing order immunization policies (SOIPs) for influenza and pneumococcal vaccinations have been found to be among the most effective strategies for increasing immunizations rates. Despite their proven efficacy these policies have not been widely adopted and there has been limited attention focused on testing particular adoption/implementation strategies. This pilot research assessed the efficacy of a minimalist strategy to implement an SOIP.

Methods: A convenience sample of 3 primary care outpatient clinics in North Carolina agreed to participate in this study and adopt and implement an SOIP for influenza and pneumococcal immunizations for their patients $\geq 65$ years old. The adoption procedure included 1-hour training for clinic nurses and providers, the provision of appropriate forms, and 2 brief reminders of protocols during the study period. Chart audits of appropriate patients who had a clinic visit during flu season (October through February) at each clinic during the baseline year of policy implementation (1999) and the year after (2000) allowed calculation of influenza and pneumococcal immunization rates as primary outcome measures.

Results: There was little evidence to indicate that these clinics made changes to implement a SOIP policy. Immunization flow sheet use, a critical process measure of SOIP implementation, was found to be less consistent than would be expected under a well-implemented SOIP. It was also found that, although influenza immunization rates did increase slightly in the 3 intervention clinics, the changes were not statistically significant. Pneumococcal immunization rate changes were also inconsistent across clinics and from baseline to post-intervention periods.

Conclusions: This minimalist effort to implement the SOIP seems not to have had sufficient impact to significantly change clinic practices. Flow sheet use, as one critical measure of SOIP implementation, did not change over the course of the intervention period. We did not find the expected increase in influenza and pneumococcal immunization rates as a result of a newly adopted SOIP. Additional research on improved strategies to fully implement SOIPs is needed to insure effective adoption of this proven systems intervention. ( $\mathrm{J}$ Am Board Fam Med 2008;21:38-44.)

Influenza and pneumococcal diseases rank as the seventh leading cause of death among the total US population, and the fifth leading cause among people $\geq 65$ years old. ${ }^{1}$ An estimated 39,400 deaths per year in the United States were attributed to influenza and pneumonia during the decade of 1990 to 1999 , with roughly $90 \%$ of them occurring among adults aged 65 or older. ${ }^{2}$ Despite these high mortality rates, only approximately one half to two thirds of seniors in the United States were vacci-

This article was externally peer reviewed.

Submitted 13 October 2006; revised 24 August 2007; accepted 6 September 2007.

From the Department of Family Medicine, University of North Carolina at Chapel Hill.

Funding: Department of Family Medicine, University of North Carolina at Chapel Hill.

Conflict of interest: none declared.

Corresponding author: George R. Gamble, PhD, 201 Vuelta Roble, Santa Fe, NM 87501 (E-mail: ggdee@QWEST.net). nated against influenza during the 2003 to $2005 \mathrm{flu}$ seasons; thus, a substantial proportion were at high risk for contracting these respiratory infections. ${ }^{3}$ Healthy People 2010 advocates $90 \%$ coverage of adults aged 65 or older for both influenza and pneumococcal vaccinations. ${ }^{4}$

In 2002, approximately $68 \%$ of North Carolinians aged 65 and older were immunized for influenza; $63 \%$ had ever been vaccinated for pneumococcal disease. $^{2}$ Efforts to increase immunizations in North Carolina, coordinated by the Adult Immunization Program in the North Carolina Department of Health and Human Services, have focused primarily on health care worker education through information packet distribution at annual association meetings and public awareness through the airing of radio Public Service Announcements during flu season [Sandy Allen, Immunization Branch, Division of Public Health, North Carolina Department of Health and Human Services, personal communication]. 
Interventions for increasing immunization rates are often categorized as client oriented (eg, mailed reminders to patients); provider oriented (eg, chart reminders to physicians); and systems oriented (eg, legislation, standing orders). ${ }^{5,6}$ Sometimes this latter group is also referred to as a mixed intervention, indicating that it often includes elements from the client- and provider-oriented interventions as well as those that are systems oriented. ${ }^{7}$ Although evidence from at least 2 systematic reviews provides a modicum of support for most immunization interventions, ${ }^{5,7}$ evidence from another systematic review strongly suggests that systems interventions, especially standing order immunization policies (SOIPs), whereby a nurse or other health care provider is authorized to administer vaccinations according to an institution- or physician-approved protocol, are among the most effective and efficient ways to increase immunization rates for pneumococcal and influenza. ${ }^{6}$ An immunization flow sheet is a critical element in this process because it is a discrete record of the patient's current immunization status. This readily accessible information greatly facilitates the assessment and administration of immunizations by the health care provider. The Advising Committee on Immunization Practices has also recommended using standing orders as an effective strategy to increase adult vaccination rates. ${ }^{8}$ Research has also shown SOIP to be effective in a variety of settings including hospitals, emergency rooms, nursing homes, and outpatient clinics. ${ }^{9-15}$

Although SOIP interventions and their effectiveness have been common knowledge for some time, they have not been widely implemented in North Carolina. We hypothesized that the cost of clinical practice change is a major obstacle that limits the diffusion of innovations like SOIPs. Indeed the issue of translating research into practice is a common theme in health care, where there is a steady stream of research supportive of various clinical innovations. ${ }^{16}$ That was the focus of this research. Here we describe one experiment to minimize the costs of adopting and implementing an SOIP in several outpatient clinics in North Carolina. Will clinics readily adopt and implement an SOIP if the "costs" of implementation are minimal?

\section{Methods}

For this research a convenience sample of community outpatient clinics within an approximate 1-hour driving distance from Chapel Hill, North Carolina were selected. Clinic criteria included a caseload of at least several hundred patients aged 65 years or older; the absence of a formal, written SOIP for influenza or pneumonia, and a willingness to adopt and implement such a policy and participate in this study. Directors of 5 outpatient clinics were invited to join in the study, 3 of whom agreed to participate. The clinics were similar in many ways. They were all relatively small (3-5 physicians) and located in the growing suburban communities in the Research Triangle area. All the clinics were associated with the same hospital in the region and they were using a paper medical record at the time of the research. This research was undertaken to test whether a minimalist implementation strategy, as defined and designed by the authors, could successfully establish a functioning SOIP and thus demonstrate a simple and effective strategy for other clinics to implement. Given that a cumbersome, complex, or costly implementation process is often viewed as a barrier to initiate change, we reasoned that a simple, low-cost implementation procedure would be an incentive for clinics to adopt an effective SOIP. Our minimalist implementation strategy included the following steps:

- Initial meeting with lead physicians and nurses who would support the policy change to discuss strategies for implementation unique to each setting.

- Sharing a model SOIP with the clinics to facilitate their own adoption of a written policy.

- Ensure that clinics have immunization flow sheets for vaccination recording and share model forms with them if necessary.

- Provide lunch to staff, especially nurses, at the beginning of the program and explain program objectives and procedures, answer questions, and assess any unexpected barriers.

- Conduct a final interview with the head nurse in each clinic at the end of the program period to learn how the policy was implemented, monitored, and followed during the course of the year.

The immunization status of eligible patients in each clinic was determined by a chart review completed by one of the investigators. To measure the change in immunization rates before and after the SOIP implementation, a chart review for the year 
before the intervention (1999) was compared with a similar review for the year of the intervention (2000). The review period was the "flu season," defined as October 1 to February 28. For both flu seasons, 1999 to 2000 and 2000 to 2001, we included all patients 65 years of age and older who had a clinic visit between October 1 and February 28 of those flu seasons. To test if there was at least an absolute increase of $15 \%$ in the influenza immunization rate ( 0.05 alpha; 0.80 power) we estimated 151 patient records would have to be reviewed in each clinic for each time period. In addition to measuring the impact of this SOIP on immunization rates, we also investigated intermediate factors to learn more about the implementation process. First, because the use of immunization flow sheets is considered to be a requisite element in the development of a standing order intervention and has been found to be closely associated with immunization rates, their use was recorded during the chart review and analysis was stratified by this factor. ${ }^{17,18}$ Secondly, because of probable protocol differences between acute care and continuity care visits, immunization rates were stratified by visit type. A medical record note that indicated a specific complaint as the purpose of the visit was classified as "acute"; routine or regular visits were considered as "continuity" visits. Finally, although an immunization flow sheet is desirable for readily accessible immunization information because it facilitates the tracking of a patient's immunization record, it is neither necessary nor sufficient to ensure that the health care provider will use the information. We therefore decided to measure the efficacy of flow sheets, comparing the proportion of immunizationeligible patients with a flow sheet who received the immunization with those without a flow sheet. Fisher's exact test was used in each of these analyses to determine whether the differences were statistically significant.

Data from the chart reviews were entered onto individual audit sheets and then were entered into an Microsoft Access database (Microsoft Corp., Redmond, WA) using a data entry screen designed to facilitate accurate, error-free entry. Immunization rates were measured as the percentage of eligible patients whose record indicated that they had received an influenza immunization during that flu season. In the case of pneumococcal immunizations, which are effective for several years, we checked for a record of a previous pneumococcal immunization. Based on the chart reviews, eligible patients were coded as either received the influenza and pneumococcal immunizations, did not receive the immunizations for some stated reason, or inadequate documentation (if no relevant information could be found in the chart). Patients who refused or, for some other explicit reason, declined an immunization were subtracted from the denominator in calculating the final immunization rates.

\section{Results}

Influenza vaccination rates

Baseline influenza vaccination rates ranged from $44 \%$ to $59 \%$ in the study clinics and increased in absolute terms from 6\% in the 1999 to 2000 period to $7 \%$ during the 2000 to 2001 period (Table 1). Although these changes were in the hypothesized direction, none reached the level of statistical significance.

\section{Pneumococcal vaccination rates}

Pneumovax immunization rates were substantially lower than the influenza immunization rates in the baseline period, with $13 \%$ to $35 \%$ in the study clinics. There were no statistically significant changes from the baseline to the postintervention period. One clinic had a slight increase, one had a slight decrease, and the third decreased markedly but with small numbers (Table 1). This latter clinic had many fewer eligible patients than were estimated at the beginning of the study, which resulted in a smaller clinic sample than had been anticipated.

\section{Type of clinic visit}

Immunization rates varied significantly by type of clinic visit. For continuity care visits, the influenza immunization rates were uniformly high (ranging from $98 \%$ to $100 \%$ ) for both the baseline and post-intervention periods, with no statistically significant changes between the 2 periods (Table 1 ). Influenza immunization rates were substantially lower for acute care visits (ranging from 6\% to $48 \%$ ), but increased from baseline to post-intervention periods in all of the clinics. In only one clinic was the increase in immunization rates for acute care visits significant (increasing from $10 \%$ to $34 \%$ ) (Table 1). Pneumovax immunization rates by type of visit followed a pattern similar to that of the influenza rates, with patients in continuity care vis- 
Table 1. Immunization Rates of Eligible Patients by Type of Visit

\begin{tabular}{|c|c|c|c|c|c|c|}
\hline & \multicolumn{2}{|c|}{1999 Flu Season } & \multicolumn{2}{|c|}{2000 Flu Season } & \multicolumn{2}{|c|}{ Change, 1999-2000 } \\
\hline & $\underset{(\mathrm{n}[\%])}{\text { Immunized }}$ & $\begin{array}{l}\text { Total } \\
\text { (n) }\end{array}$ & $\underset{(\mathrm{n}[\%])}{\operatorname{Immunized}}$ & $\begin{array}{l}\text { Total } \\
\text { (n) }\end{array}$ & $\begin{array}{c}\text { Change } \\
(\%)\end{array}$ & $\begin{array}{c}\text { Fisher's Exact } \\
\text { Test }\end{array}$ \\
\hline \multicolumn{7}{|l|}{ Influenza } \\
\hline \multicolumn{7}{|l|}{ All visits } \\
\hline Clinic 1 & $87(58.8)$ & 148 & $98(65.3)$ & 150 & 6.5 & NS \\
\hline Clinic 2 & $14(48.3)$ & 29 & $24(54.5)$ & 44 & 6.3 & NS \\
\hline Clinic 3 & $64(43.8)$ & 146 & $78(51.3)$ & 152 & 7.5 & NS \\
\hline \multicolumn{7}{|c|}{ Continuity visits } \\
\hline Clinic 1 & $83(100.0)$ & 83 & $92(95.80)$ & 96 & -4.2 & NS \\
\hline \multicolumn{7}{|l|}{ Clinic $2^{*}$} \\
\hline Clinic 3 & $55(98.2)$ & 56 & $41(97.60)$ & 42 & -0.6 & NS \\
\hline \multicolumn{7}{|l|}{ Acute visits } \\
\hline Clinic 1 & $4(6.2)$ & 65 & $6(11.10)$ & 54 & 5.0 & NS \\
\hline Clinic 2 & $14(48.3)$ & 29 & $24(54.50)$ & 44 & 6.3 & NS \\
\hline Clinic 3 & $9(10.0)$ & 90 & $37(33.60)$ & 110 & 23.6 & SIG \\
\hline \multicolumn{7}{|l|}{ Pneumovax } \\
\hline \multicolumn{7}{|l|}{ All visits } \\
\hline Clinic 1 & $12(16.4)$ & 73 & $14(17.9)$ & 78 & 1.5 & NS \\
\hline Clinic 2 & $7(35.0)$ & 20 & $5(18.5)$ & 27 & -16.5 & NS \\
\hline Clinic 3 & $13(13.5)$ & 96 & $12(13)$ & 92 & -0.5 & NS \\
\hline \multicolumn{7}{|c|}{ Continuity visits } \\
\hline Clinic 1 & $10(33.3)$ & 30 & $12(28.60)$ & 42 & -4.8 & NS \\
\hline \multicolumn{7}{|l|}{ Clinic $2^{*}$} \\
\hline Clinic 3 & $11(33.3)$ & 33 & $6(21.40)$ & 28 & -11.9 & NS \\
\hline \multicolumn{7}{|l|}{ Acute visits } \\
\hline Clinic 1 & $2(4.7)$ & 43 & $2(5.60)$ & 36 & 0.9 & NS \\
\hline Clinic 2 & $7(35.0)$ & 20 & $5(18.50)$ & 27 & -16.5 & NS \\
\hline Clinic 3 & $2(3.2)$ & 63 & $6(9.40)$ & 64 & 6.2 & NS \\
\hline
\end{tabular}

*Insufficient number of patients.

NS, not significant; SIG, significant.

its having a higher immunization rate (ranging from $33 \%$ to $65 \%$ ) than those in acute visits (ranging from $3 \%$ to $35 \%$ ) (Table 1). The changes in the rates between the baseline and post-intervention periods were not statistically significant.

\section{Flow Sheets}

Although all clinics reported using flow sheets in 1999 for the baseline period, flow sheets were found in only $11 \%$ to $61 \%$ of patients' medical charts in the study clinics (Table 2). The clinics had a single flow sheet that included both influenza and pneumovax sections. We coded this sheet according to the presence of recorded information in both of the sections. Thus, both a medical record in which no flow sheet was found and one in which there was a flow sheet but no data entered in either place was coded as "no flow sheet present." A flow sheet that had data entered in either or both sections was coded accordingly. All clinics increased their use of flow sheets from that baseline period to the post-intervention period for influenza-eligible patients, but the changes were not statistically significant (Table 2).

To assess the efficacy of the flow sheets, we compared the immunization rates of patients with and without flow sheets. The flu immunization rate for those who had a flow sheet in their medical chart was quite high in both time periods, ranging from $93 \%$ to $100 \%$ (Table 2). For eligible patients without flow sheets, the comparable rate was $<9 \%$ for all the clinics in both time periods. Slight increases in the immunization rate from the baseline to postintervention periods were not statistically significant. The proportion of pneumococcaleligible patients with a flow sheet receiving a pneu- 


\begin{tabular}{|c|c|c|c|c|c|c|}
\hline & \multicolumn{2}{|c|}{1999 Flu Season } & \multicolumn{2}{|r|}{2000 Flu Season } & \multicolumn{2}{|c|}{ Change, 1999-2000 } \\
\hline & $\begin{array}{l}\text { Total patients } \\
\text { (n) }\end{array}$ & $\begin{array}{l}\text { Patients with flow sheet } \\
(\%[\mathrm{n}])\end{array}$ & $\begin{array}{l}\text { Total } \\
\text { (n) }\end{array}$ & $\begin{array}{l}\text { Patients with flow sheet } \\
(\%[\mathrm{n}])\end{array}$ & $\begin{array}{c}\text { Change } \\
(\%)\end{array}$ & $\begin{array}{c}\text { Fisher's Exact } \\
\text { Test }\end{array}$ \\
\hline \multicolumn{7}{|c|}{ Patients vaccinated for: } \\
\hline \multicolumn{7}{|c|}{ Influenza } \\
\hline Clinic 1 & 151 & $60.90(92)$ & 151 & $67.50(102)$ & 6.60 & NS \\
\hline Clinic 2 & 30 & $50.00(15)$ & 45 & $51.10(23)$ & 1.10 & NS \\
\hline Clinic 3 & 156 & $40.40(63)$ & 163 & $44.20(72)$ & 3.80 & NS \\
\hline \multicolumn{7}{|l|}{ Pneumovax } \\
\hline Clinic 1 & 93 & $61.60(151)$ & 85 & $56.30(151)$ & -5.30 & NS \\
\hline Clinic 2 & 13 & $43.30(30)$ & 21 & $46.70(45)$ & 3.40 & NS \\
\hline \multirow[t]{2}{*}{ Clinic 3} & 17 & $10.90(156)$ & 12 & $7.40(163)$ & -3.50 & NS \\
\hline & & $\begin{array}{l}\text { Patients immunized } \\
\qquad \%[\mathrm{n}])\end{array}$ & & $\begin{array}{l}\text { Patients immunized } \\
\text { (\% [n] }\end{array}$ & & \\
\hline \multicolumn{7}{|c|}{ Patients with flow sheets } \\
\hline \multicolumn{7}{|c|}{ Influenza } \\
\hline Clinic 1 & 92 & $93.5(86)$ & 102 & $95.1(97)$ & 1.6 & NS \\
\hline Clinic 2 & 15 & $93.3(14)$ & 23 & $100.0(23)$ & 6.7 & NS \\
\hline Clinic 3 & 63 & $98.4(62)$ & 72 & $97.2(70)$ & -1.2 & NS \\
\hline \multicolumn{7}{|l|}{ Pneumovax } \\
\hline Clinic 1 & 93 & $12.9(12)$ & 85 & $12.9(11)$ & 0.0 & NS \\
\hline Clinic 2 & 13 & $46.2(6)$ & 21 & $19.0(4)$ & -27.2 & NS \\
\hline Clinic 3 & 17 & $64.7(11)$ & 12 & $75.0(9)$ & 10.3 & NS \\
\hline \multicolumn{7}{|c|}{ Patients without flow sheets } \\
\hline \multicolumn{7}{|c|}{ Influenza } \\
\hline Clinic 1 & 59 & $1.7(1)$ & 49 & $2.0(1)$ & 0.3 & NS \\
\hline Clinic 2 & 15 & $0.0(0)$ & 22 & $4.5(1)$ & 4.5 & NS \\
\hline Clinic 3 & 93 & $2.2(2)$ & 91 & $8.0(8)$ & 6.6 & NS \\
\hline \multicolumn{7}{|l|}{ Pneumovax } \\
\hline Clinic 1 & 58 & $0.0(0)$ & 66 & $4.5(3)$ & 4.5 & NS \\
\hline Clinic 2 & 17 & $5.9(1)$ & 24 & $4.2(1)$ & -1.7 & NS \\
\hline Clinic 3 & 139 & $1.4(2)$ & 151 & $2.0(3)$ & 0.5 & NS \\
\hline
\end{tabular}

mococcal shot was quite variable among the clinics, ranging from $13 \%$ to $65 \%$ in 1999 (Table 2). Those patients without flow sheets had rates below $6 \%$. The change in efficacy from 1999 to 2001 was also variable and not statistically significant.

During the end-of-project interview with the head nurse in each of the intervention clinics we learned that, although none of them had a formal, written SOIP before this intervention year, they had been emphasizing the importance of immunizations, particularly influenza, for their elderly and high-risk patients over the past few years. This emphasis included systematic protocols to give influenza vaccinations to at-risk patients and use of immunization flow sheets (head nurses, personal communication, 2003).

\section{Discussion}

Our hypotheses that an SOIP could be introduced and effectively implemented in outpatient clinics with minimal effort and cost and have immediate immunization rate improvements of at least $15 \%$ were not substantiated in this study. First and most significantly, based on findings from statistical analyses and interviews with the head nurses in all the clinics, we concluded that the SOIP was not implemented in the test clinics.

Because of the extant systematic immunization practices for high-risk patients, of which we were unaware at the beginning of the study, the nurses did not perceive the need for changing their routines when the "new" written SOIP was put into 
place. They simply viewed the new SOIP policy as a "paper change" and they continued to do what they always did.

The explanation that there was little if any behavioral change on the part of the nursing staff during the intervention period was also supported by evidence that there was little change in the proportion of patients who had immunization flow sheets in their charts from the baseline to postintervention periods. Because universal flow sheet usage is the requisite and key element for implementing an SOIP, this evidence indicates that nursing staff did not change their use of flow sheets to implement the new SOIP. The use of flow sheets has also been found to be associated with higher vaccination rates in other studies. ${ }^{17,18}$

We also believe that this minimalist strategy was ineffective because individual and institutional routines and practices are more cumbersome to change than this minimalist effort assumes. Even though the SOIP seems to be a simple policy, it is complex when it comes to implementation. Observational studies that have looked at the immunization process in clinics have documented the complex range of operational factors that are associated with successful vaccination policies and procedures. ${ }^{19}$ The failure to appreciate the complexity of this process “. . may explain why so many demonstrably efficacious interventions identified by the US Task Force on Community Preventive Services fail to be routinely implemented and why immunization rates have remained static over the past decade."19

To some extent our finding that virtually all the patients who came in for continuity visits were immunized but only a low percentage of those who came in for a acute care visits were immunized highlights this complexity issue. It seems that all the study clinics have made immunizations routine in their continuity care practices, as evidenced by their virtually universal immunization of those patients; however, that routine does not currently include immunizations for acute care visits. Similar findings have been reported by other studies. ${ }^{18}$ At least one study has reported that it is feasible to systematically give influenza and pneumovax immunizations to acute care patients, for example in an emergency department, but the effort required "intense supervision." 12 Our minimalist strategy would not qualify in any way as including that level of effort.
Although the SOIP provides an excellent guideline for systematically improving immunization rates, it seems as though more substantial training and/or monitoring and supervising activities would be necessary to achieve a fuller implementation of the policy and larger and more consistent immunization changes, which seem to be feasible for an SOIP.

This study's major limitation was the mistaken premise that the sample clinics did not have any systemic influenza vaccination policy before our intervention. Rather than simply asking if they had a written SOIP we should have inquired about their procedures for giving immunizations and then discussed changes that would be necessary to implement the SOIP. This inquiry into current circumstances before any discussion of the desired change is often considered a fundamental procedural step in any behavioral change situation.

The introduction and adoption of an SOIP can be an important step to increasing immunization rates, but the results from this study highlight the critical importance of the entire implementation and monitoring process in making this policy effective. A strategy or tool can only be as effective as its implementation and use. There are many ways clinics might implement this policy, but this minimalist model may not yield sufficient results. The results from this pilot study should prove useful in planning the development of strategies to disseminate standing order interventions in a variety of health care networks, including private practice settings. SOIPs are simply, but the clinic environment into which it is introduced is a complex one that changes only when the providers and management are motivated to make those changes. Putting research into practice is at best a formidable process that requires planning, training, implementation, and monitoring. A minimalist intervention model might be successful if coupled with monitoring and implementation in an environment motivated to make and ready to embrace change. Both monitoring and motivation seems to have been very limited in our test clinics.

\section{References}

1. Centers for Disease Control and Prevention. National Vital Statistics Report. Deaths: leading causes for 2002; 2005;53:17.

2. Centers for Disease Control and Prevention. Public health and aging: influenza vaccination coverage 
among adults aged $\geq 50$ years and pneumoccocal vaccination coverage among adults aged $\geq 65$ yearsUnited States, 2002. MMWR 2003;52:987-92.

3. Centers for Disease Control and Prevention. Estimated influenza vaccination coverage among adults and children-United States, September 1-November 30, 2004. MMWR 2004;53:1147-53.

4. US Department of Health and Human Services. Healthy People 2010, objectives 14,29a and 14, 29b. Washington, DC: US Department of Health and Human Services, Public Health Service; 2000.

5. Grimshaw JM, Shirran L, Thomas R, et al. Changing provider behavior: an overview of systematic reviews of interventions. Med Care 2001;39(8 suppl 2):III -45 .

6. Gyorkis TW, Tannenbaum TN, Abrahamowicz M, et al. Evaluation of effectiveness of immunization delivery methods. Can J Pub Health 1994;85:S1-29.

7. Sarnoff, R, Rundall, T. Meta-analysis of effectiveness of interventions to increase influenza immunization rates among high-risk population groups. Med Care Res Rev 1998;55:432-56.

8. Centers for Disease Control and Prevention. Use of standing orders programs to increase adult vaccination rates: recommendations of the Advisory Committee on Immunization Practices. MMWR 2000; 49:15-26.

9. Crouse BJ, Nichol K, Peterson DC, Grimm MB. Hospital-based strategies for improving influenza vaccination rates. J Fam Pract 1994;38:258-61.

10. Nichol KL, Korn JE, Margolis KL, Poland GA, Petzel RA, Lofgren RP. Achieving the national health objective for influenza immunization: success of an institution-wide vaccination program. Am J Med 1990;89:156-60.
11. Slobodkin D, Zielske PG, Kitlas JL, McDermott MF, Miller S, Rydman R. Demonstration of the feasibility of emergency department immunization against influenza and pneumococcus. Ann Emerg Med 1998;32:537-4.

12. Slobodkin D, Kitlas J, Zielske P. Opportunities not missed-systematic influenza and pneumococcal immunization in a public inner-city emergency department. Vaccine 1998;16:1795-802.

13. Nichol KL, Grimm MB, Peterson DC. Immunizations in long-term care facilities: policies and practice. J Am Geriatr Soc 1996;44:349-55.

14. Nichol KL. Ten-year durability and success of an organized program to increase influenza and pneumococcal vaccination rates among high-risk adults. Am J Med 1998;105:385-92.

15. Margolis KL, Nichol KL, Wuorenma J, Von Sternberg TL. Exporting a successful influenza vaccination program from a teaching hospital to a community outpatient setting. J Am Geriatr Soc 1992;40: 1021-3.

16. Bradley EH, Webster TR, Baker D, et al. Translating research into practice: speeding the adoption of innovative health care programs. Issue brief \#724. New York: The Commonwealth Fund; 2004.

17. Nowalk MP, Zimmerman RK, Cleary SM, Bruehlman RD. Missed opportunities to vaccinate older adults in primary care. J Am Board Fam Pract 2005; 18:20-7.

18. Nowalk MP, Zimmerman RK, Feghali J. Missed opportunities for adult immunization in diverse primary care office settings. Vaccine 2004;22:3457-63.

19. Fontanesi J, Shefer AM, Fishbein DB, et al. Operational conditions affecting the vaccination of older adults. Am J Prev Med 2004;26:265-70. 